Results Abstract PTU-254 table 1 outlines GPs' confidence in managing gastroenterological conditions while Abstract PTU-254 table 2 outlines their rating of service developments in chronic disease management, referral pathways and other services. GPs commented on the need for "clear referral pathways" and "rapid access clinics" outside of the 2-Week Rule remit. They wanted "clear plans" for shared care with "rapid access if problems" as well as "support from nurse specialists" and "access to telephone advice" for both patients and themselves. GPs wanted "workload implications to be recognised" and "money to follow the patient" if more patients are managed in primary care.

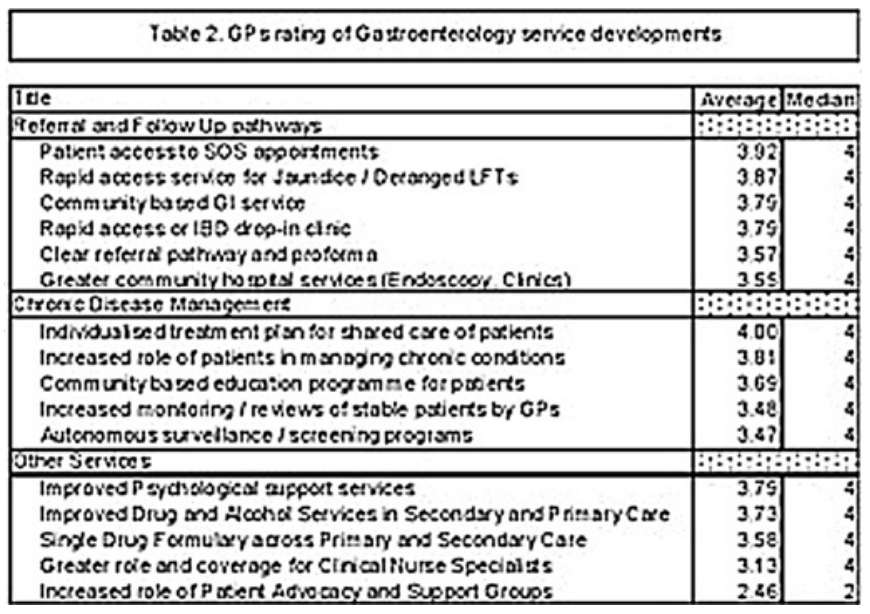

Conclusion The survey has identified which conditions GPs are confident managing in primary care and those which need additional support from secondary care. Future service development is needed in areas of chronic disease management, referrals pathways and allied services. GPs value rapid access to secondary care as well as patient access to SOS appointments and nurse specialists. Developing local pathways, such as with Map of Medicine, can help with referrals and managing chronic conditions in primary care. Online surveys are an easy way to ask GPs about their own confidence in managing gastroenterological conditions as well as their opinion on service developments.

Competing interests None declared.

\section{PTU-255 IS THE SERVICE PROVISION OF DIRECT ACCESS UPPER GI ENDOSCOPY BEING USED EFFECTIVELY BEYOND 2010?}

doi:10.1136/gutjnl-2012-302514c.255

${ }^{1} \mathrm{~S}$ Mahmood, ${ }^{*}$ S S Singh, ${ }^{1} \mathrm{M}$ Naseer, ${ }^{1} \mathrm{~S}$ Sarkar. ${ }^{1}$ Department of Gastroenterology, Royal Liverpool University hospital, Liverpool, UK; ${ }^{2}$ Department of Medicine, Royal Liverpool University hospital, Liverpool, UK

Introduction Upper GI Endoscopy can generate significant income through payment by results (PBR) in the UK. Primary care physicians have a direct access for endoscopy via open access (GPOA) or 2 -week rule (2WR) systems and appropriateness of these lists have been long-debated. With the current financial cut backs within the NHS, it is worthwhile to re-evaluate their effectiveness.

Aim To assess the effectiveness of GPOA/2WR upper GI Endoscopy service.

Methods Retrospective audit of GPOA and 2WR lists between April and October 2011 was performed. 483 referrals were audited $(n=400$ $2 \mathrm{WR}, \mathrm{n}=83 \mathrm{GPOA}$ ) for patient demographics, indications, significant diagnosis, additional tests and patient outcomes.

Results 2WR-Patients: Audit capture rate $86 \%$. Median age of the patient was 65 years (range 18-96), 58\% were Females, with median ASA 2. NICE criteria for referrals was not met in $12 \%$ and a further $14 \%$ had a gastroscopy within 3 years. Outcomes: Significant diagnoses (cancer, oesophagitis, Barretts, PUD, Coeliac Disease, Stricture, Helicobacter pylori gastritis) were present in $44 \%$ (cancer diagnosis $3.4 \%$ ). An endoscopy urease test was performed in $48 \%$, with a $30 \%$ positivity rate. Biopsies were taken in $64 \% .86 \%$ were discharged back to the GP, $4 \%$ were referred to clinic, $6 \%$ booked for a repeat endoscopy and $4 \%$ referred to cancer MDT. GPOA Patients: Audit capture rate was $96 \%$ referrals. Patients were younger than the 2WR with median age 52 years (range 17-86) $(p=0.0001)$, and healthier with median ASA 1 ( $p=0.0001) .53 \%$ were females. $31 \%$ of patients did not meet NICE guidelines and $4 \%$ had a gastroscopy within 3 years. Outcomes: Significant diagnoses were made in $29.9 \%$ (cancer diagnosis $1.2 \%$ ). While the cancer diagnoses with $2 \mathrm{WR}$ was comparable $(\mathrm{p}=0.2)$, there were less benign diagnoses $(p=0.01)$. Additional tests included urease test in $19 \%$, positivity rate $20 \%$ and biopsies $14 \%$. Patient outcomes were similar to 2WR with $88 \%$ of patients discharged directly back to GP, $6 \%$ booked for a repeat endoscopy, 4\% referred to Gastroenterology Clinic and $2 \%$ to a cancer MDT. Overall, 51 patients (12\%) had a previous endoscopy within 3 years, none of which had cancer and 83 patients $(19 \%)$ did not meet NICE guidelines that is, $31 \%$ were inappropriate.

Conclusion With $31 \%$ of patients being inappropriate and low cancer pick up rate, the value of these lists may be questionable. However benign pathology was significant in both lists and therefore process mapping the patient to see if the endoscopy changed the patient's management would be useful given the very high discharge rate to the GP. There still remains significant room for improving the effective use of these services.

Competing interests None declared.

\section{PTU-256 OPTIMISING BOWEL SCREENING COLONOSCOPY UPTAKE WITH NURSE-LED TELEPHONE ASSESSMENT CLINICS}

doi:10.1136/gutjnl-2012-302514c.256

S Osmond. * Aneurin Bevan Health Board, Caerphilly, UK

Introduction Bowel Screening Wales utilises nurse-led telephone assessments pre-colonoscopy for screening participants following a positive faecal occult blood test (FOBt) result. This approach has been shown to optimise colonoscopy uptake.

Methods A literature review was carried out to determine the cost effectiveness and efficiency of telephone assessment as indicated by overall screening colonoscopy appointment uptake. Nurse-led telephone assessment is an advanced practice. Nurses undertaking this form of assessment should have undergone suitable training and supervision, be competent in their practice and accountable for their actions. Advanced assessment is the detailed, systematic collection of relevant information about the patient's problems and health status which requires a specialist knowledge, skill and extensive experience to uncover the relevant information being given and discard the irrelevant. ${ }^{1}$ Bowel Screening Wales was introduced nationally in October 2008 and operates from a single hub which is responsible for inviting participants, processing completed kits and providing results for participants. There are 13 Local Assessment Centres (LAC) which have a responsibility to provide endoscopy, pathology and radiology services for participants who have had a positive FOBt result. Specialist Screening Practitioners are based in each LAC. In Wales each Health Board covers a large geographical area therefore telephone assessments are a more effective method of pre-colonoscopy assessment in terms of cost, time and travel requirements. It has also been found that this approach results in a lower colonoscopy default rate due to improved participant involvement in the decision making process. 
Results Data published in the Bowel Screening Wales First Round Report dated 29 September 2011 shows that 6493 (82.2\%) of SSP appointments were completed by telephone. $0.5 \%$ of participants required or requested a face to face assessment. $90 \%$ of participants assessed were fit for colonoscopy of which $89.4 \%$ attended a procedure. The $10 \%$ of participants found not fit were offered an alternative to colonoscopy such as CT colonography.

Conclusion The literature reviewed clearly shows that telephone assessment for Bowel Screening Wales participants following a positive FOBt is an effective and acceptable form of information gathering and provision. Good attendance rates for assessment appointments and colonoscopy procedures are evidenced by the Bowel Screening Wales first round report.

Competing interests None declared

\section{REFERENCE}

1. McGee $\mathbf{P}$, ed. Advanced assessment and differential diagnosis. Advanced Practice in Nursing and the Allied Health Professions. Oxford: Wiley-Blackwell, 2009.

\section{PTU-257 "TRIALS AND TRIBULATIONS OF SETTING UP AN ANAESTHETIST LED DAY-CASE ENDOSCOPY LIST"}

doi:10.1136/gutjnl-2012-302514c.257

${ }^{1} \mathrm{~S}$ V Murugesan, ${ }^{*} \mathrm{M}$ Davies, ${ }^{1} \mathrm{~N}$ Haslam, ${ }^{1} \mathrm{H} \mathrm{L}$ Smart, ${ }^{1} \mathrm{~S}$ Sarkar. ${ }^{1}$ Department of Gastroenterology, Royal Liverpool and Broadgreen University Hospitals NHS Trust, Liverpool, UK; ${ }^{2}$ Department of Anaesthetics, Royal Liverpool and Broadgreen University Hospitals NHS Trust, Liverpool, UK

Introduction Endoscopy in UK is performed under conscious sedation in daycase (DC) endoscopy units. Patients intolerant of the procedure subsequently undergo the procedure under a general anaesthetic in operating theatres. However whether this service could be effectively offered in a "non-operating theatre room" setting through an anaesthetist-led sedation service (ALS) is not well established within the UK.

Aim To evaluate a new provision of an ALS in a day case endoscopy unit.

Methods Service evaluation audit (June-August 2011) of a new weekly dedicated ALS at the Royal Liverpool Hospital was performed. Administrative, anaesthetic and case records and satisfaction scores ( 1 =unsatisfactory; $5=$ fully satisfied; patients, anaesthetists, endoscopists) and "patient journey" was evaluated.

Results Administration: Three cases were scheduled per listed. Waiting time was a median 9 weeks compared to a median 6 weeks for other lists. Of the 25 patients listed, only 20 (80\%) had a procedure. DNA rate was $16 \%$ and cancellation rate $4 \%$. Patients and Indication: Median age was 53 years (range 18-80 years), median ASA was 2 (range 1-3), 8 (42\%) were female. 20 underwent propofol assisted endoscopy (7 colonoscopy, 8 HALO $^{\circledR}$, 3 ERCP, 1 OGD and 1 EUS). Procedural details: ALS was provided by a consultant anaesthetist assisted by operating theatre personnel and endoscopic procedures by a consultant gastroenterologist. All were successfully completed (mean duration of $33 \mathrm{~min}$, range 20-70 min). Modification of anaesthetic equipment was required in all but one and additional equipment had to be borrowed in all. Adverse events were minor (rate 10\%)-transient hypoxia (not required ventilation) and hypotension. All patients were discharged successfully as DC. 7-day readmissions and 30-day mortality was 0 . The median satisfaction score was 5 (range $4-5$ ) for patients, 5 (range 4-5) for anaesthetists and 5 (3-5) for endoscopists. The additional cost for provision of such a service included the services of the anaesthetist (one programmed activity) and OTP and for drugs (propofol)
Conclusion Deep sedation for selected endoscopic procedures can be safely and efficiently provided through a dedicated anaesthetist-led list in a remote endoscopy unit. However, waiting times were longer than other lists and a significant number of patients DNA'd/ cancelled. We are now setting up pre-assessment clinics and increasing the number of lists to improve the service further.

Competing interests None declared.

\section{PTU-258 A UK NATIONAL COMPARATIVE AUDIT OF APPROPRIATENESS OF RED CELL TRANSFUSION IN MEDICAL PATIENTS}

doi:10.1136/gutjnl-2012-302514c.258

${ }^{1} \mathrm{~S}$ Coda, ${ }^{*} \mathrm{~K}$ Pendry, ${ }^{2} \mathrm{~T}$ Davies, ${ }^{3} \mathrm{~J}$ Grant-Casey, ${ }^{4} \mathrm{~J}$ Wallis, ${ }^{5} \mathrm{C}$ Taylor, ${ }^{6} \mathrm{~B}$ Astbury, ${ }^{7} \mathrm{E}$ Hughes, ${ }^{8} \mathrm{~J}$ Reid, ${ }^{9} \mathrm{M}$ Horan, ${ }^{10} \mathrm{~T}$ Davies, ${ }^{1} \mathrm{~A} V$ Thillainayagam. ${ }^{1}$ Department of Gastroenterology, Imperial College Healthcare NHS Trust, London, UK; ${ }^{2}$ NHS Blood and Transplant, Manchester, UK; ${ }^{3}$ NHS Blood and Transplant, Oxford, UK; ${ }^{4}$ Newcastle upon Tyne Hospitals NHS Foundation Trust, Newcastle upon Tyne, UK; ${ }^{5}$ The Dudley Group of Hospitals, Dudley, UK; ${ }^{6}$ Wrexham Maelor Hospital, Wrexham, UK; ${ }^{7}$ Betsi Cadwaladr University Health Board, Gwynedd, UK; ${ }^{8}$ University Hospitals Leicester NHS Trust, Leicester, UK; ${ }^{9}$ University of Manchester, Manchester, UK; ${ }^{10}$ Central Manchester University Hospitals NHS Foundation Trust, Manchester, UK

Introduction The decrease in red cell usage (15\%) that has been achieved in recent years has mainly been achieved through the use of blood conservation strategies adopted for surgical patients. Despite national guidelines for blood transfusion being available, agreed triggers for transfusion still need to be established in clinical practice, together with better decision-making in anaemia management to further reduce inappropriate transfusion of red cells in medical patients.

Methods The main aim of this audit was to observe the extent of inappropriate red cell transfusions, taking account of relevant clinical information and pretransfusion assessment. In autumn 2011, a total of 8759 red cell transfusions in medical units from almost all UK hospitals ( $>90 \%$ ) were audited in 3 selected weeks. Through an algorithm based on clinical and diagnostic information provided, a panel of expert physicians estimated the appropriateness of the transfusion. Subgroup analysis was also undertaken to identify patients with a reversible cause of anaemia in whom timely diagnosis and treatment of the original condition would have almost certainly prevented the recourse to blood transfusion.

Results Preliminary analysis has revealed many cases of red cell transfusion when the pretreatment haemoglobin $(\mathrm{Hb})$ was within acceptable limits. The findings show that $4.1 \%$ cases had a pretransfusion $\mathrm{Hb}$ higher than $10 \mathrm{~g} / \mathrm{dl}$ (median 7.8, range 2.3-17.7) and $5.8 \%$ had a posttransfusion $\mathrm{Hb}$ of greater than $12 \mathrm{~g} / \mathrm{dl}$ (median 9.9, range 4.2-19.3). Serum ferritin was measured in only $38 \%$ of cases and of these $9.3 \%$ of patients had a ferritin $<15 \mu \mathrm{g} / \mathrm{l}$. Of all the transfusion episodes, only 1748 were for blood loss, while 180 were for prophylaxis prior to a procedure. The vast majority were for anaemia, with the remaining 48 reports not having enough data. In blood loss, the commonest indication was haematemesis (1029 cases), while 423 were primarily for rectal bleeding.

Conclusion This national comparative audit shows: (i) there is substantial inappropriate red cell transfusion in medical patients; (ii) gastrointestinal bleeding is far and away the major reason for transfusion in the setting of blood loss among medical patients. The frequent use of red cell transfusion in the absence of clinical necessity seems to derive from the use of above-threshold triggers, and suboptimal management of anaemia with a reversible cause.

Competing interests None declared. 